\title{
Analysis of the heavy alkali element post deposition treatment: which factors determine the electronic structure and transport properties of the hetero-junction in CIGS thin film solar cells
}

Shiqing Cheng ${ }^{\dagger}$, Kaizhi Zhang ${ }^{\dagger} a$, Shuping Lin ${ }^{a}$, Yunxiang Zhang ${ }^{a}$, Yun Sun ${ }^{a}$, Wei $\operatorname{Liu}^{a *}$

a Institute of Photoelectronic Thin Film Devices and Technology of Nankai University, Key Laboratory of Photoelectronic Thin Film Devices and Technology of Tianjin, Engineering Research Center of Thin Film Photoelectronic Technology, Ministry of Education, Tianjin 300350, P.R. China

* E-mail: wwl@,nankai.edu.cn

$\dagger$ Theses authors contributed equally to this work 


\section{Supporting Information:}
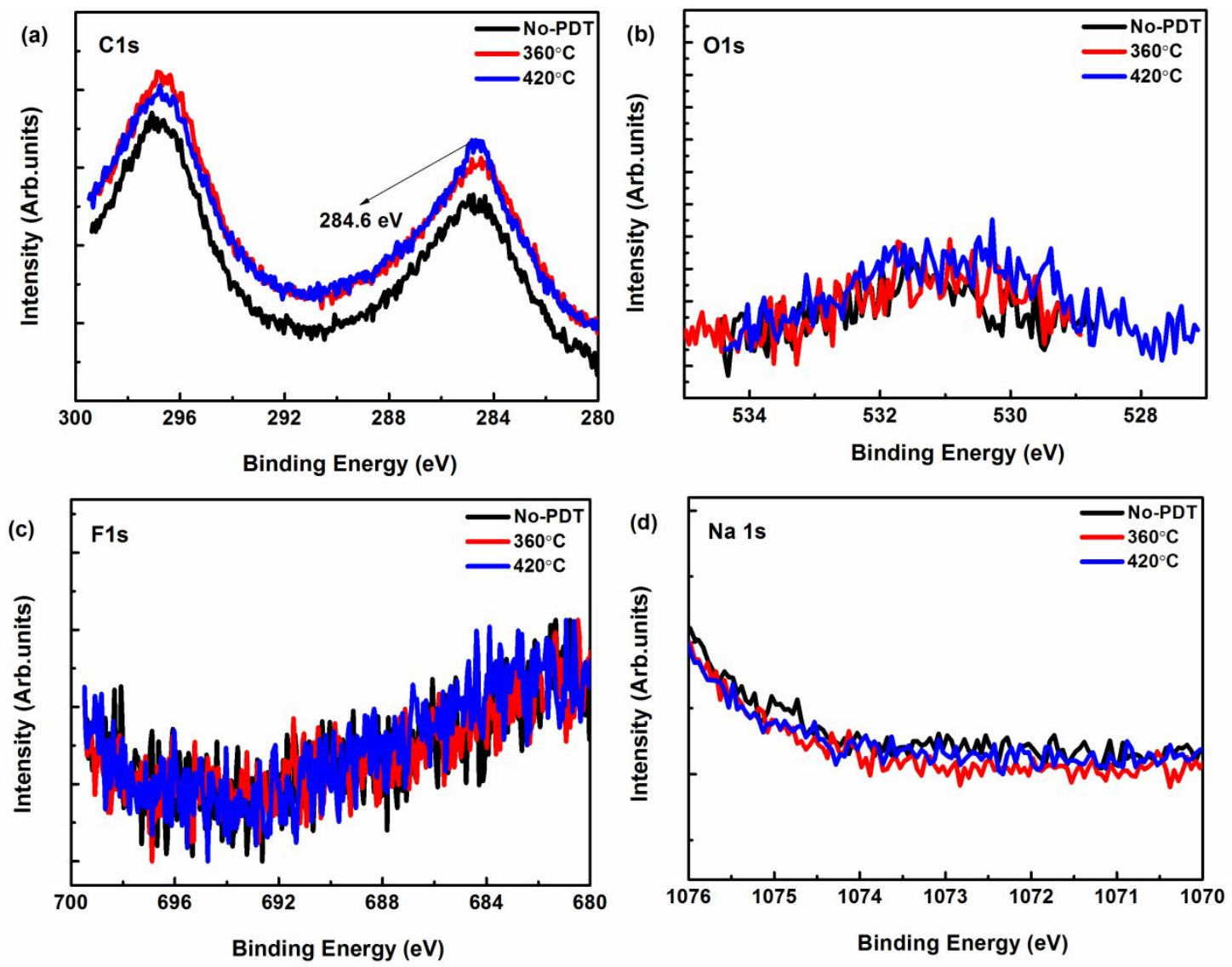

Figure S1. The photo emission peaks of (a) C 1s, (b) O 1s, (c) F 1s, (d) Na 1s . 

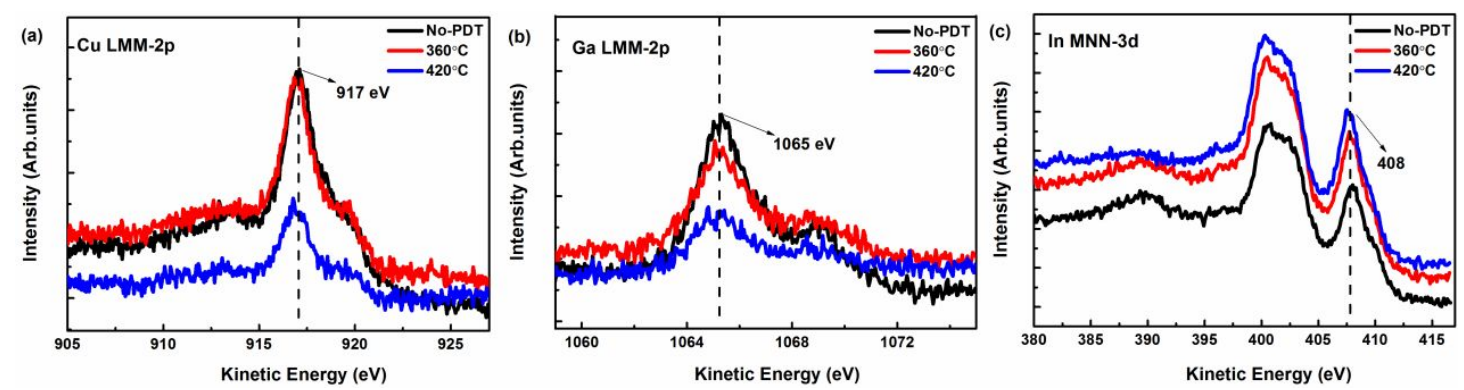

Figure S2. The Auger spectra of the Cu LMM-2P (a), Ga LMM-2P (b) and In MNN-3d (d). The Kinetic energy of the Cu LMM-2P, Ga LMM-2P and In MNN-3d peaks are $917 \mathrm{eV}, 1065 \mathrm{eV}$ and $408 \mathrm{eV}$ respectively. 